\title{
AVALIAÇÃO DA TERAPÊUTICA CIRÚRGICA DO DIVERTÍCULO FARINGOESOFÁGICO PELA SUTURA MECÂNICA
}

\author{
Pharyngoesophageal diverticulum surgical therapy evaluation using linear stapling device \\ José Luis Braga de AQUINO, Marcelo Manzano SAID, José Francisco Salles CHAGAS, \\ Vânia Aparecida LEANDRO-MERHI, Eusébio José GALLO-JUNIOR, \\ Gustavo Nardini CECCHINO, Mayra Todeschini ASSUNÇÃO
}

\begin{abstract}
Trabalho realizado nos Serviços de Cirurgia Torácica e de Cabeça e Pescoço do Hospital Celso Pierro da Faculdade de Medicina da Pontifícia Universidade Católica de Campinas, Campinas, SP, Brasil.
\end{abstract}

DESCRTORES - Divertículo faringoesofágico. Divertículo de Zenker. Suturas
RESUMO - Racional - O divertículo faringoesofágico apresenta-se como distúrbio pouco frequente e é provável que este fato seja responsável pela dificuldade encontrada na escolha do método terapêutico mais eficaz. O tratamento cirúrgico ainda é a principal opção terapêutica. No entanto, há controvérsias quanto à técnica cirúrgica que garante melhores resultados. Objetivo - Avaliar a eficácia da diverticulectomia com miotomia do músculo cricofaríngeo pela sutura mecânica linear no tratamento cirúrgico do divertículo faringoesofágico em uma série de pacientes no tocante às suas complicações locais e sistêmicas. Método - Foram atendidos 19 pacientes com divertículo de Zenker. Todos apresentavam as condições clínicas necessárias e preenchiam os critérios de elegibilidade para realização do procedimento cirúrgico. Após a diverticulectomia com sutura mecânica todos foram submetidos a avaliação pós-operatória no sentido de identificar a manifestação de qualquer tipo de complicação. Os pacientes foram estudados retrospectivamente através da análise de prontuários e descrição da internação hospitalar realizada no momento da operação e da evolução ambulatorial. Resultados - A totalidade apresentou resultados satisfatórios, sem evidência de fístula ao nível da sutura da faringe no período pós-operatório precoce. Somente dois indivíduos perderam seguimento na avaliação tardia e um desenvolveu estenose faríngea revertida com dilatação endoscópica. Todos referiram estar satisfeitos com o procedimento empregado e relataram melhora na qualidade de vida ao final do seguimento, proporcionada pelo retorno da deglutição. Conclusão - A diverticulectomia com a miotomia do músculo cricofaríngeo e posterior fechamento da faringe com sutura mecânica linear mostrou-se técnica eficaz, com poucas complicações pósoperatórias e melhora significativa na qualidade de vida.

\section{Correspondência:}

José Luis Braga de Aquino,

e-mail:jlaquino@sigmanet.com.br

Fonte de financiamento: não há

Conflito de interesses: não há

Recebido para publicação: Aceito para publicação:

HEADINGS - Esophagopharyngeal diverticulum. Zenker's diverticulum. Surgical stapler.
ABSTRACT - Background - Pharyngoesophageal diverticulum presents itself as an uncommon disorder. Thus, choosing the most effective treatment method may be challenging. Surgical treatment remains as the main option. However, establishing the best surgical technique is still controversial. Aim - To evaluate the results of diverticulecomy with chricopharyngeal miotomy and linear stapler closure of the pharynx in a patient sample with Zenker's diverticulum regarding local and systemic complications. Methods - Nineteen patients with pharyngoesophageal diverticulum were admitted. All of them presented the clinical conditions required to indicate the surgical procedure. Patients were evaluated with regard to any post-operative complications. This study was conducted retrospectively with patients' data analysis. Results - Patients showed satisfactory results, with no evidence of fistula at the level of the pharyngeal suture. Two out of 19 lost post-operative follow-up and one of them had pharyngeal stenosis reverted with endoscopic dilation. The entire sample reported being satisfied with the procedure emphasizing improvement of the quality of life mainly due to the return of physiological deglutition. Conclusion - The diverticulectomy with myotomy and posterior pharyngeal closure with linear surgical stapler proved to be an effective technique, offering a lower risk of post-operative complications and improving the overall quality of life. 


\section{INTRODUÇÃO}

A pesar das datas serem um pouco divergentes entre os estudos publicados, o divertículo faringoesofágico foi inicialmente descrito por Abraham Ludlow em 176415; porém só em 1877 essa doença foi bem estudada pelo patologista alemão Albert Zenker ${ }^{24}$, o qual correlacionou os aspectos clínicos e anatomopatológicos, conferindo seu nome a ela. No entanto a primeira ressecção de um divertículo de Zenker realizada com sucesso só veio a ocorrer em 1886 por Whealer ${ }^{1}$.

Ele consiste basicamente em uma deformação sacular dilatada, localizada na parede posteroinferior da mucosa faríngea, acima do esfíncter esofágico superior, sobre uma região situada entre as fibras musculares estriadas oblíquas do músculo constritor inferior da faringe e as fibras transversas do músculo cricofaríngeo, denominada por sua vez de Triângulo de Killian. Essa região encontra-se mais predisposta à herniação da mucosa em virtude da alta pressão intraluminal sobre uma área vulnerável, na qual as fibras musculares encontram-se mais escassas, expondo por sua vez a mucosa hipofaríngea ${ }^{1,24}$.

O divertículo faringoesofágico apresenta-se como doença pouco frequente entre a população, sendo responsável por $1-3 \%$ das queixas de disfagia além de representar cerca de $4 \%$ dos doentes com afecção do esôfago ${ }^{1,18}$. Sua prevalência é mais significativa na faixa etária compreendida entre a sexta e a oitava década de vida, com o pico de incidência por volta dos 70 anos de idade, sendo rara antes dos quarenta ${ }^{1,18}$. Isso se deve ao fato da perda do tônus muscular e diminuição da resistência da parede posterior que ocorre fisiologicamente com o envelhecimento. Predomina no sexo masculino na proporção de três homens para cada mulher ${ }^{2}$. Sua ocorrência é mais marcante nos países do norte da Europa, sendo relativamente raro nos países do extremo oriente. Poucos são os estudos que apontam a ocorrência exata do divertículo de Zenker nos países da América do Sul, incluindo o Brasil, mas sabe-se que não é doença frequente entre a população ${ }^{1,2,18}$.

$O$ paciente portador manifesta disfagia e regurgitação como principais sintomas, podendo apresentar halitose e perda de peso como sintomas secundários, o que compromete de maneira significativa sua qualidade de vida18,20,22.

O diagnóstico pode ser realizado através de investigação clínica, complementada pela realização de exames radiográficos contrastados baritados da faringe e esôfago, além da visualização direta do esôfago através da endoscopia digestiva alta. Eventualmente faz-se necessário o uso da manometria ${ }^{1,16}$.

O tratamento é fundamentalmente cirúrgico, com diverticulectomia ou diverticulopexia, seguido de miotomia do músculo cricofaríngeo, embora nos últimos anos, alguns autores advogam o tratamento endoscópico ${ }^{1,14,16,19}$. Apesar da diverticulectomia ser um procedimento padronizado, não é isenta de complicações, das quais notavelmente se destaca a fístula cervical com consequente deiscência da sutura no nível da faringe ${ }^{13,18}$. Na maioria das vezes esta complicação é resolvida com tratamento conservador, porém há grande comprometimento na qualidade de vida do paciente, uma vez que retarda a deglutição, o que pode resultar em indesejável comprometimento nutricional.

Apósoadventodasuturamecânica, demonstrandose segura e precisa, foi amplamente empregada em vários segmentos do trato gastrointestinal, seja em afecções benignas ou malignas ${ }^{4,5}$. Esse tipo de sutura evidenciou a possibilidade de minimizar as complicações ao nível das anastomoses, pois é efetuada em dois planos e de modo invertido, o que reduz a isquemia e necrose tecidual ${ }^{5}$.

Pelo pouco destaque que tem sido dado ao uso da sutura mecânica linear no fechamento da faringe após a ressecção do divertículo faringoesofágico, optou-se pela utilização deste tipo de sutura em uma série de pacientes submetidos a diverticulectomia com a miotomia do músculo cricofaríngeo.

O objetivo deste estudo foi avaliar a eficácia desta modalidade cirúrgica no tocante às suas complicações locais e sistêmicas.

\section{MÉTODO}

\section{Casuística}

De junho de 1999 a dezembro de 2010 foram atendidos 19 pacientes no Serviço de Cirurgia Torácica do Hospital e Maternidade Celso Pierro - PUC Campinas, SP, Brasil sendo que todos apresentavam divertículo faringoesofágico com indicação para realização de diverticulectomia com miotomia do músculo cricofaríngeo seguido de sutura mecânica linear. Os pacientes foram esclarecidos a respeito do procedimento em questão, quanto ao prognóstico, riscos e objetivos do tratamento e o procedimento foi efetuado somente após o consentimento dos mesmos.

Nesta série avaliada retrospectivamente houve predominância do sexo masculino, representado por 16 pacientes $(84,2 \%)$, perante três do sexo feminino $(15,7 \%)$, com idade entre 62 e 77 anos (média de 69,5 anos). Deste grupo de indivíduos, 15 deles eram tabagistas (78,9\%), consumindo uma média de 20 cigarros/dia em tempo variável de 35 a 54 anos. Além disso, $13(68,4 \%)$ referiram ser etilista de uma dose de destilado/dia com tempo variável de 25 a 47 anos.

\section{Avaliação pré-operatória}

O diagnóstico foi feito através da observação e avaliação clínica, radiológica e endoscópica. $\mathrm{Na}$ avaliação clínica foram observados sintomas de disfagia leve intermitente para sólidos em todos os pacientes, com tempo variável de dois a cinco anos. Quanto aos 
demais sintomas, oito pacientes $(42,1 \%)$ apresentavam regurgitação associada à tosse e três $(15,7 \%)$ referiram eructações frequentes além de emagrecimento com perda de cerca de $5 \%$ do peso corporal total .

Estudo radiográfico contrastado confirmou a presença do divertículo faringoesofágico em todos os indivíduos (Figura 1).

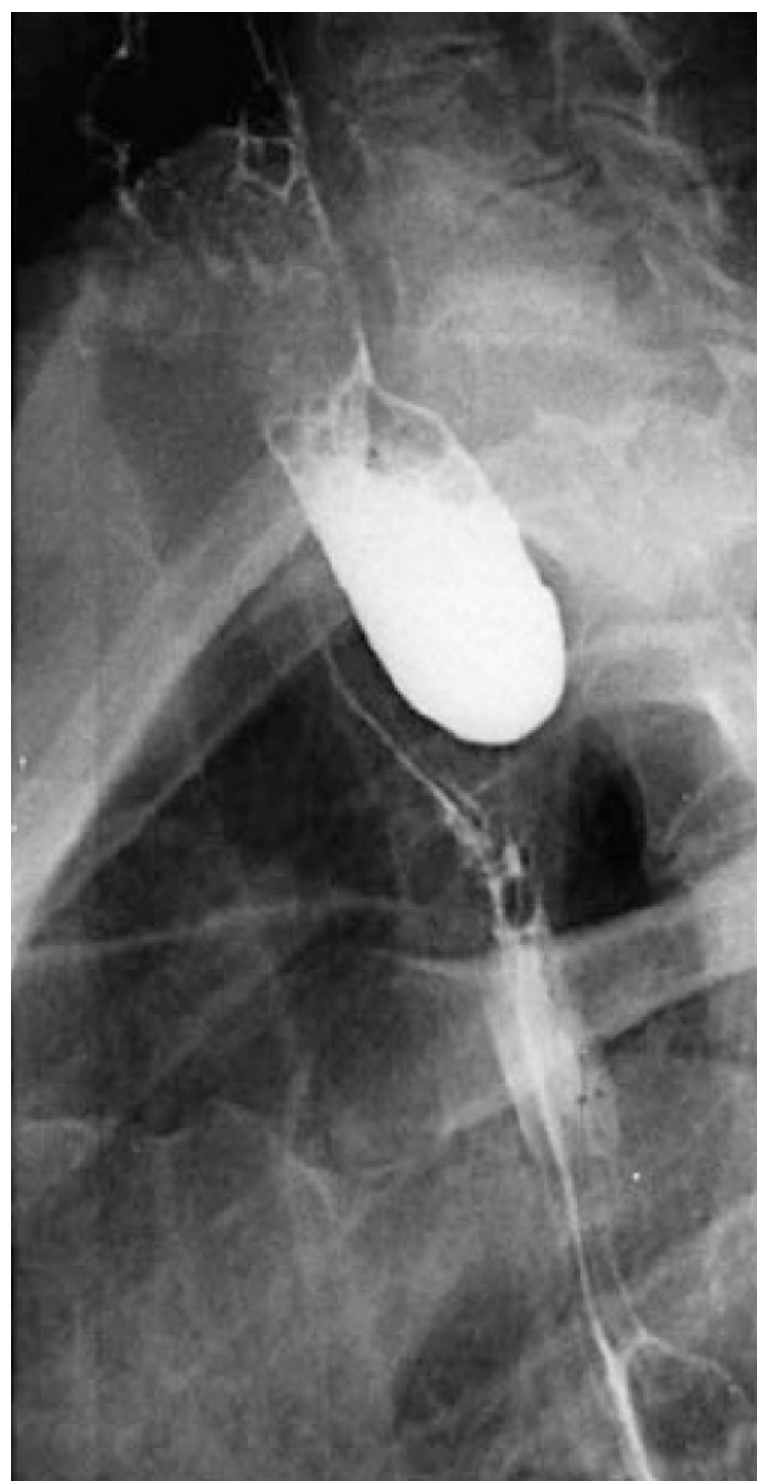

FIGURA 1 - Esofagograma evidenciando o divertículo faringoesofágico

A endoscopia digestiva alta evidenciou em todos os pacientes a presença de divertículos de grandes dimensões, sendo as mesmas compreendidas entre 5 a $9 \mathrm{~cm}$ de diâmetro. Deve-se ressaltar que esse exame também avaliou que não havia a presença de doença associada ao divertículo em nenhum dos pacientes.

Em todos os sujeitos a avaliação clínica e nutricional pré-operatória demonstrou-se satisfatória, o que permitiu a realização do procedimento cirúrgico proposto.

\section{Técnica cirúrgica e avaliação pós-operatória}

A técnica cirúrgica realizada foi a diverticulectomia com a miotomia do músculo cricofaríngeo de acordo com a seguinte padronização: a) incisão cervical supraclavicular esquerda e descolamento dos retalhos de pele; b) exposição do músculo esternocleidomastoideo esquerdo e dissecção da sua porção medial com exposição da faringe e esôfago cervical; c) identificação do divertículo e sua dissecção das estruturas adjacentes até o momento de exposição do seu colo junto à parede da faringe; d) secção do divertículo e fechamento da faringe com sutura mecânica linear pelo aparelho TA $45 \mathrm{~mm}$; e) miotomia do músculo cricofaríngeo até o esôfago cervical proximal em extensão de $3 \mathrm{~cm}$; f) colocação de sonda nasogástrica para alimentação no pós-operatório imediato; g) colocação do dreno laminar por contraabertura lateral e fechamento da incisão por planos.

A avaliação pós-operatória foi focada: a) nas complicações sistêmicas - notadamente de origem cardiovascular, respiratória ou infecciosa - sendo ela avaliação baseada na observação da evolução clínica diária dos pacientes e pelos resultados dos exames laboratoriais e de imagem que eram solicitados quando se tornavam necessários; e b) nas complicações locais, no que se refere à estenose e principalmente à deiscência da sutura da faringe, com consequente fistulização.

A deiscência da sutura da faringe com fístula foi diagnosticada através da observação clínica e confirmada pela visualização direta de saída de secreção salivar pela região cervical até $05^{\circ}$ dia de pós-operatório. $\mathrm{Na}$ ausência de evidência clínica de fístula na anastomose, foi realizado no $5^{\circ}$ dia faringoesofagograma para observar se houve extravasamento do contraste. No caso de resultado negativo foi liberada a dieta líquida via oral, evoluindo progressivamente para as formas pastosas e sólidas, de acordo com a aceitação do paciente.

Em relação à estenose da sutura da faringe, o diagnóstico foi eminentemente clínico, orientado pela disfagia a partir do $30^{\circ}$ dia de pós-operatório e diminuição do diâmetro da anastomose, evidenciada pela radiografia contrastada e endoscopia digestiva alta.

Finalmente, a perspectiva da qualidade de vida individual também foi avaliada, principalmente em relação ao retorno da deglutição adequada após a intervenção cirúrgica e, nos casos em que se constatou disfagia, foi avaliado o grau, classificando-a quanto sua intensidade em leve (alimentos sólidos), moderada (alimentos pastosos) ou intensa (alimentos líquidos).

\section{RESULTADOS}

\section{Avaliação precoce}

Em avaliação precoce realizada nos primeiros 30 dias de pós-operatório, dois pacientes $(10,5 \%)$ apresentaram infecção pulmonar com resposta 
satisfatória observada a partir da introdução de antibioticoterapia específica. Tais pacientes eram os mais idosos da série, tabagistas de longa data e portadores de doença pulmonar obstrutiva crônica.

Uma vez que nenhum indivíduo apresentou quadro sugestivo de fistulização com saída de secreção digestiva pelo dreno ou pela incisão cervical, procedeuse a realização de faringoesofagograma contrastado no $5^{\circ}$ dia de pós-operatório para avaliar a viabilidade da anastomose. O exame radiográfico contrastado não evidenciou extravasamento do contraste ao nível da sutura da faringe, demonstrando assim que não houve a ocorrência da fistula cervical na totalidade dos casos. A partir desse período, foi liberada a dieta oral, inicialmente para líquidos, com evolução adequada e gradativa até atingir deglutição para alimentos de consistência pastosa e sólido, sendo que todos os pacientes apresentaram uma boa aceitação da mesma.

Não houve óbito nesta série.

\section{Avaliação tardia}

A avaliação a médio e longo prazo foi realizada em 17 pacientes, uma vez que houve perda de seguimento clínico de dois pacientes.

O tempo de avaliação variou entre dois meses a nove anos de pós-operatório (média 4,9 anos), sendo que esses pacientes foram avaliados ambulatorialmente a cada três meses no primeiro ano e, a partir do segundo ano de pós-operatório, semestralmente.

Durante o seguimento ambulatorial, um paciente apresentou disfagia para sólidos a partir do $60^{\circ}$ dia de pós-operatório. A endoscopia digestiva alta e $\mathrm{O}$ faringoesofagograma contrastado revelaram estenose ao nível da sutura da faringe, sendo na ocasião indicado dilatação endoscópica com boa evolução após três sessões. Tanto este paciente como os demais estudados nesse período declararam-se satisfeitos com o procedimento cirúrgico realizado, pois apresentaram o retorno deglutição fisiológica, evoluindo com ganho ponderal adequado e melhora significativa na qualidade de vida.

\section{DISCUSSÃO}

A ocorrência do divertículo faringoesofágico é pouco frequente no nosso meio de tal forma que poucos serviços possuem um número expressivo de pacientes, suficientes para proporcionar experiência satisfatória no seu manuseio e tratamento, fato que também explica a limitada casuística deste estudo.

O tratamento desta afecção é fundamentalmente cirúrgico e baseado na sua etiopatogenia, de tal modo que a maioria dos autores tem preconizado diverticulectomia seguida da miotomia do músculo cricofarínge $\mathrm{O}^{1,8,9,23}$. Outros preconizam a diverticulopexia associada à miotomia do cricofaríngeo, demonstrando que os resultados são semelhantes quando comparados com a diverticulectomia e miotomia ${ }^{12,13,18}$. Outra indicação menos comum para ressecção do divertículo de Zenker baseia-se no princípio de prevenir a transformação maligna, podendo ser forma de diagnosticar ainda na fase precoce um provável carcinoma in situ ${ }^{3}$.

Sabe-se que recentemente a indicação da diverticulopexia fica restrita a pacientes selecionados, como idosos, com comorbidades clinicas de alto risco e com divertículos de pequena dimensões, geralmente menores de $3 \mathrm{~cm}^{10,12,13}$.

A opção por indicar esta técnica cirúrgica se fez com base na avaliação endoscópica realizada em todos os pacientes que evidenciou dimensões iguais ou maiores que $5 \mathrm{~cm}$. Além disso, a média de idade dos indivíduos estudados era 69,5 anos e nenhum deles apresentava comorbidades clínicas relevantes.

Atualmente o tratamento endoscópico do divertículo faringoesofágico também possui muitos adeptos e exige experiência. Esta técnica consiste em dividir o septo entre o divertículo e o esôfago sob controle endoscópico ${ }^{7,21}$. Van Overbbek ${ }^{21}$ relata os resultados do tratamento endoscópico em 545 pacientes ao longo de 30 anos, obtendo melhora satisfatória da disfagia em $91 \%$ deles, com reduzidas taxas de complicações. Ishioka et al. ${ }^{11}$ relatam sua experiência utilizando fibroendoscópio para realizar a secção do septo em 42 doentes com divertículo de Zenker, obtendo bons resultados com 7,1\% de recidiva da disfagia.

Em relação às complicações da diverticulectomia, a fístula cervical consequente à deiscência da sutura da faringe tem sido relatada em uma incidência variável de 5-35\% 1,13,17,18,22. Apesar desta complicação na maioria das vezes ser resolvida com tratamento conservador, com curativos diários e suporte nutricional com sonda nasoenteral, ela compromete de forma significativa a qualidade de vida do paciente por retardar o retorno da deglutição por via oral.

Por esses e outros motivos tem sido demonstrado vantagem na utilização da sutura mecânica, que por ser dupla e invertida, ela favorece melhor coaptação das bordas da sutura e minimiza a ocorrência de deiscência. Este fato também pôde ser observado neste estudo, já que nenhum dos pacientes apresentou deiscência da sutura ao nível da faringe. Bonavina et al. ${ }^{6}$ também enfatizaram as vantagens do uso da sutura mecânica no fechamento da faringe após ressecção do divertículo, pois dos 116 pacientes submetidos a este procedimento, nenhum deles evoluiu com fístula cervical.

Por se atuar, na maioria das vezes, em pacientes idosos e com potencial comorbidades, principalmente da esfera cardiopulmonar, pressupõem-se que possam ocorrer complicações sistêmicas no pós-operatório, fato este presente em $10,5 \%$ desta série e similar a de outros estudos ${ }^{1,13,17,18,23}$. O tabagismo é outro fator relevante no prognóstico, pois os dois pacientes que apresentaram infecção pulmonar, ambos eram 
portadores de pneumopatias crônicas secundárias a um tabagismo de longa data.

$\mathrm{Na}$ avaliação realizada a longo prazo, vários autores têm demonstrado que a diverticulectomia com a miotomia do cricofaríngeo proporciona o desaparecimento dos sintomas da disfagia na maioria dos pacientes ${ }^{1,10,12,14,17,18,20,23}$. Andreollo et al. ${ }^{1}$, avaliando 38 pacientes submetidos ao tratamento cirúrgico do divertículo de Zenker com tempo médio de seguimento de 14 meses, enfatizam as vantagens da diverticulectomia com miotomia do cricofaríngeo, pois o grupo que foi submetido a este procedimento obteve resultado excelentes em $84,6 \%$ para $66,6 \%$ do grupo em que foi realizado a diverticulopexia e miotomia.

No seguimento médio de 4,8 anos de pósoperatório dos pacientes desta série, foi demonstrado que o procedimento cirúrgico realizado ofereceu vantagens, sendo que somente um paciente apresentou pequena estenose ao nível da faringe, resolvida precisamente com três sessões de dilatação. Deve-se ressaltar que todos os pacientes conseguiram resgatar de modo adequado a sua deglutição, obtendo dessa forma significativa melhora em sua qualidade vida.

\section{CONCLUSÃO}

A diverticulectomia com a miotomia do músculo cricofaríngeo e posterior fechamento da faringe com sutura mecânica linear mostrou-se técnica eficaz, com poucas complicações pós-operatórias e melhora significativa na qualidade de vida.

\section{REFERÊNCIAS}

1. Andreollo NA, Lopes LR, Brandalise NA, Camargo MA, Coelho Neto JS. Tratamento cirurgico do divertículo de Zenker: Diverticulopexia versus Diverticulectomia. ABCD Arq Bras Cir Dig 2007;20(4):245-9

2. Andreollo NA, Lopes LR, Brandalise NA. Distúrbios motores do esôfago.In : Endoscopia digestiva $.1^{\text {a }}$ Edição. Rio de Janeiro: Sobed, Medsi 1994, p 58-68

3. Aquino JLB, Said MM, Bordalo R. Carcinoma in situ em Diverticulo Faringo Esofagico. Rev Col Bras Cir. 2005;32(2):106-107

4. Aquino JLB, Said MM, Brandi LA, Oliveira JMP, Maziero D, Leandro Merhi V. Tubo gástrico isoperistaltico de grande curvatura gástrica com sutura mecânica no tratamento cirurgico do câncer de esôfago irressecavel. ABCD Arq Bras Cir Dig 2009;229(3):147-52
5. Aquino JLB. Avaliação da anastomose esofagogástrica cervical com sutura mecânica em pacientes com megaesôfago avançado. Rev Col Bras Cir 2005;3:143-146

6. Bonavina L, Bona D, Abraham M, Saino G, Abate E. Long term results of endosurgical and open surgical aprroach for Zenker diverticulum. World j Gastroenterl 2007;13(8):2568-9

7. Dohlman G, Mattson O. The endoscopic operation for hypopharingeal diverticula. A roentgencinematografic study. Arch Otolaryngol 1960;71:744-52

8. Ellis FH, Schlegel JF, Lynch VP, Payne WS. Crycopharyngeal myotomy for Pharyngoesophageal diverticulum. Ann Surg 1969;170:340-9

9. Gullane PJ, Willet JM, Heeneman H. Zenker's diverticulum. J Otolaryngol 1983; 12:53-57

10. Gutschow CA, Hamoir M, Rombaux P, Otte JB, Goncette L, Collard JM. Management of pharyngoesophageal (Zenker's) diverticulum: which technique? Ann Thorac Surg 2002;74(5): 1677-82

11. Ishioka I, Sakai P, Maluf Filho F. Endoscopic incision of Zenker's diverticula. Endoscopy 1995;27:433-37

12. Laccourreye $O$, Menard M, Cauchois R, Huart J, Jouffre V, Brasnu D et al. Esophageal diverticulectomy. Laryngoscope 1994;104:889-92

13. Leporrier J, Salame $E$, Gignoux M, Segol P. Zenker's diverticulum:diverticulopexy versus diverticulectomy. Ann Chir 2001;126(1):42-5

14. Lerut T, Van Raemdonck D, Guelinckx P, Dom R, Geboes K. Zenker's diverticulum: is amyotomy of the crycopharyngeus useful? How long should it be? Hepatogastroenterology 1992;39:127-31

15. Ludlow A. A case of obstructed deglutition, from a preter natural dilatation of and bag formed in the pharynx. Med Soc Phys 1767;3:35-101

16. Migliore $M$, Payne $H$, Jeyasingham K. Pathophysiologic Basis for peration on Zenker's Diverticulum. Ann Thorac Surg 1994;57:1616-21

17. Okano N, Vargas EC, Moriya T, Carneiro JJ, Elias-Junior AM. Diverticulo do esôfago: analise de 24 pacientes portadores do divertículo de Zenker. Acta Cir Bras 2000;15(2):45-49

18. Ribeiro GBS, Mielke F, Volkweis BS, Schirmer CC, Kruel CDP, Morellato $G$. Tratamento cirurgico do diverticulo de Zenker. ABCD Arq Bras Cir DIG 2008;21(3):110-3

19. Sakai P. Endoscopic treatment of Zenker's diverticulum. Gastrointest Endosc 2007:65(7):1054-5

20. Simic A, Radovanovic N, Stojakov D, Bjelovic M, Kotarac M, Sabljak P. Surgical experience of the national institution in the treatment of Zenker's diverticula. Acta Chir Iugosl 2009; 56(1):25-33

21. Van Overbeek JJM. Meditation on the pathogenesis of hypoharyngeal( Zenker's) diverticulum and a report of endoscopic treatment in 545 patients.Ann Otol Rhinol Laryngol 1994;103:178-185

22. Villasennor G, Lorenzo L. Tratamiento quirúrgico de los transtornos motores Del esôfago. Cir Gen 2007;29:253-9

23. Wittrick IJ, Gullane PJ, Yeung E. Outcome analysis of Zenker's diverticulectomy and cricopharyngeal myotomy. Head \& Neck 1995;17:382-88

24. Zenker FA, Von Ziemssen H. Krankheiten des Oesophagus.. In: Von Ziemssen $\mathrm{H}$, ed Hankbuch der Speciellen Pathologie und Therapie, vol 7(suppl). Leipzig: FCW Vogel, 1877: 1-87 